\title{
Gaussian Quadrature of Integrands Involving the Error Function
}

\section{By J. P. Vigneron and Ph. Lambin}

\begin{abstract}
Orthogonal polynomials corresponding to the weight function $1-\operatorname{erf}(x)$ and defined on the positive real axis are constructed. Abscissas and weight factors for the associated Gaussian quadrature are then deduced (up to 12-point formulas). The stability of the algorithm used for this particular computation is discussed. An example is provided to test the efficiency of the new Gaussian rule.
\end{abstract}

1. Introduction. In recent years, the field of automatic quadrature has achieved important progress. For tabulated functions with arbitrary grid spacing, cubic spline integrators supply an efficient way to obtain an approximation to a definite integral [1]. In the case where very little is known about the integrand, adaptive quadrature methods [2] can be used with a high probability of success. When possible, however, Gaussian formulas [3] remain extremely interesting, regarding the few integrand evaluations needed. This advantage is especially desirable when the integrand computation is very time consuming. Rather few weight functions and intervals of integration have been considered so far [3]-[5]. In the present paper, a Gaussian quadrature formula is derived for computing expressions of the form

$$
I=\int_{0}^{\infty} \operatorname{erfc}(x) f(x) d x
$$

where

$$
\operatorname{erfc}(x)=1-\frac{2}{\sqrt{\pi}} \int_{0}^{x} e^{-t^{2}} d t
$$

is the complementary error function [4], and $f(x)$ is a regular function.

To determine the abscissas $x_{i}$ and weight factors $w_{i}$ appearing in the Gaussian expression

$$
I \approx \sum_{i=1}^{n} w_{i} f\left(x_{i}\right)
$$

it is necessary to obtain the set of orthogonal polynomials $p_{k}(x), k=0,1, \ldots, n$, corresponding to the following scalar product

$$
(f, g)=\int_{0}^{\infty} \operatorname{erfc}(x) f(x) g(x) d x
$$


The construction of these polynomials and the derivation of the integration formula is treated in Section 2.

2. Orthogonal Polynomials and Gaussian Formula. The Schmidt orthogonalization procedure can be used to generate these polynomials from the set of nonorthogonal functions

$$
1, x, x^{2}, x^{3}, \ldots, x^{n}
$$

This procedure is described in [6] and amounts to determining recursively the polynomials $p_{k}(x)$ through the following relation

$$
\begin{gathered}
p_{0}(x)=1, \\
p_{k}(x)=x^{k}-\sum_{n=0}^{k-1} \frac{\int_{0}^{\infty} \operatorname{erfc}(t) t^{k} p_{n}(t) d t}{\int_{0}^{\infty} \operatorname{erfc}(t)\left[p_{n}(t)\right]^{2} d t} p_{n}(x),
\end{gathered}
$$

for $k=1,2, \ldots, n$.

If the explicit expression for these polynomials

$$
p_{k}(x)=\sum_{j=0}^{k} p_{j}^{k} x^{j}
$$

is introduced in (6) and (7), the following relation is obtained

$$
\sum_{n=0}^{k} p_{n}^{k} x^{k}=x^{k}-\sum_{n=0}^{k-1} \frac{\sum_{i=0}^{n} p_{i}^{n} \mu_{i+k}}{\sum_{l=0}^{n} \sum_{i=0}^{n} p_{i}^{n} p_{l}^{n} \mu_{i+l}} \sum_{j=0}^{n} p_{j}^{n} x^{j}
$$

where the quantities $\mu_{p}$ are the moments of the weight function

$$
\mu_{p}=\int_{0}^{\infty} \operatorname{erfc}(x) x^{p} d x=\frac{\Gamma(p / 2+1)}{\sqrt{\pi}(p+1)} .
$$

In Eq. (9), the right-hand side can be put under an explicit polynomial form, by reordering the summation on $n$ and $j$. This leads to the following recursion relations for computing the coefficients $p_{j}^{k}$ of the orthogonal polynomials

$$
p_{k}^{k}=1
$$

and, for $n \neq k$,

$$
p_{n}^{k}=-\sum_{j=n}^{k-1} \frac{\sum_{i=0}^{j} p_{i}^{j} \mu_{i+k}}{\sum_{i=0}^{j} \sum_{l=0}^{j} p_{i}^{j} p_{l}^{j} \mu_{i+l}} p_{n}^{j}
$$

3. Discussion. A large amount of comments exists in literature on the illconditioned character of the problem of determining the zeros and weights for Gaussian rules [7]-[10]. It is not obvious, in a general case, to forecast the stability of relation (12). This question has in fact two different aspects. If one considers the 
moments $\mu_{k}$ as exactly known quantities, the recursion scheme can propagate the truncation error that affects the coefficients $p_{j}^{k}$ at each stage of the recursive process. On the other hand, the polynomial coefficients may be sensitive to errors introduced in computing the moments $\mu_{p}$. Both effects act to progressively reduce the accuracy of the computed coefficients. Furthermore, the evaluation of the zeros of the polynomials and the subsequent computation of the weight factors will introduce further errors. For this reason, an arithmetic of about 35 figures has been used in performing the computation realized here and a check on the $\mu$-wise sensitivity of the polynomial coefficients, as well as the abscissas and weight factors, has been completed.

\section{TABLE 1}

Sensitivity of the 12th-degree orthogonal polynomial coefficients to a slight variation of the moments $\mu_{p}$. A relative increase of $3 \cdot 10^{-33}$ of all moments has been performed. $\Delta p_{12}^{i} / p_{12}^{i}$ is the relative change of the polynomial coefficients, $\Delta x_{i} / x_{i}$ is the relative change of its zeros, and $\Delta w_{i} / w_{i}$ the corresponding relative change in the weight factor.

\begin{tabular}{|c|c|c|c|c|c|c|}
\hline $\mathrm{i}$ & $\frac{\Delta p_{12}^{\mathrm{i}}}{\mathrm{p}_{12}^{\mathrm{i}}}$ & & $\frac{\Delta x_{i}}{x_{i}}$ & & $\frac{\Delta w_{i}}{w_{i}}$ & \\
\hline 1 & $(-16)$ & 3.4 & $(-17)$ & 3.7 & $(-17)$ & 3.6 \\
\hline 2 & $(-16)$ & 3.0 & $(-17)$ & 3.7 & $(-17)$ & 2.9 \\
\hline 3 & $(-16)$ & 2.7 & $(-17)$ & 3.6 & $(-17)$ & 1.4 \\
\hline 4 & $(-16)$ & 2.4 & $(-17)$ & 3.3 & $(-17)$ & 1.1 \\
\hline 5 & $(-16)$ & 2.1 & $(-17)$ & 3.1 & $(-17)$ & 4.7 \\
\hline 6 & $(-16)$ & 1.8 & $(-17)$ & 2.9 & $(-17)$ & 9.9 \\
\hline 7 & $(-16)$ & 1.6 & $(-17)$ & 2.7 & $(-16)$ & 1.7 \\
\hline 8 & $(-16)$ & 1.2 & $(-17)$ & 2.5 & $(-16)$ & 2.5 \\
\hline 9 & $(-16)$ & 1.0 & $(-17)$ & 2.4 & $(-16)$ & 3.5 \\
\hline 10 & $(-17)$ & 7.3 & $(-17)$ & 2.2 & $(-16)$ & 1.8 \\
\hline 11 & $(-17)$ & 4.8 & $(-17)$ & 2.1 & $(-16)$ & 6.6 \\
\hline 12 & $(-17)$ & 2.4 & $(-17)$ & 2.0 & $(-16)$ & 8.6 \\
\hline 13 & & 0.0 & & & & \\
\hline
\end{tabular}




\section{TABLE 2}

First sixteen polynomials orthogonal with respect to the scalar product $(f, g)=\int_{0}^{\infty} \operatorname{erfc}(x) f(x) g(x) d x$. The coefficients are ordered following the decreasing powers of $x$. For example, the second-degree polynomial is approximately $p_{2}(x)=x^{2}-1.35 x+0.264$.

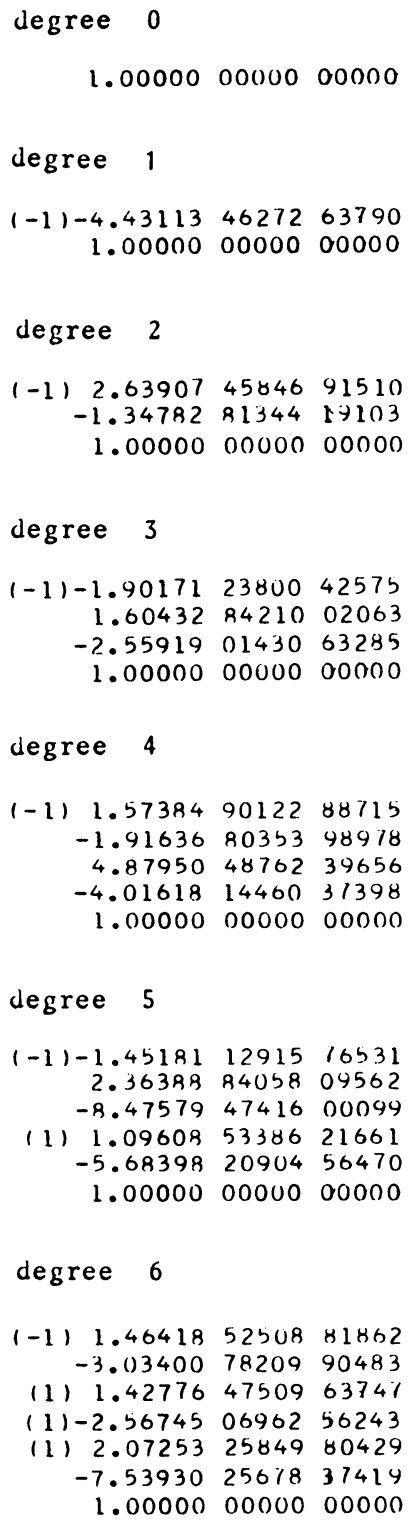

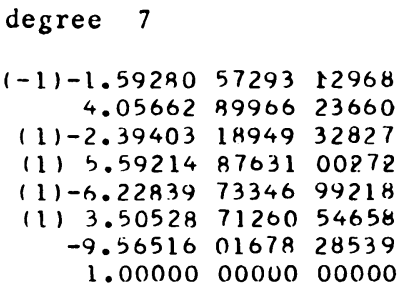


TABLE 2 (continued)

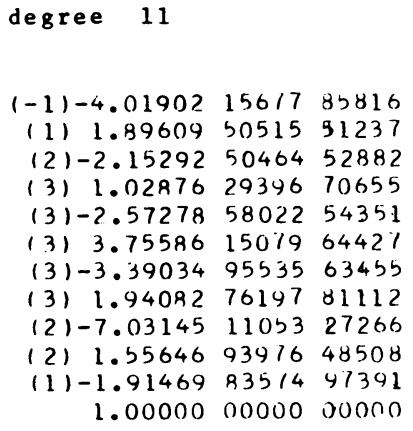

degree 12

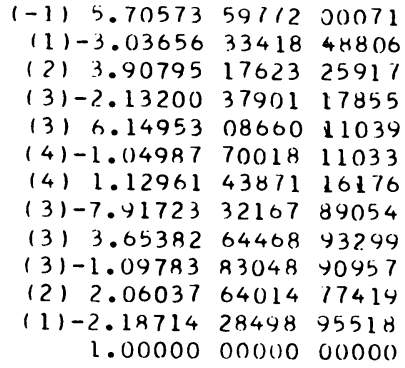

TABLE 3

Abscissas and weight factors for the 2-point to 12-point Gaussian integration of $\int_{0}^{\infty} \operatorname{erfc}(x) f(x) d x \approx \sum_{i=1}^{n} w_{i} f\left(x_{i}\right)$.

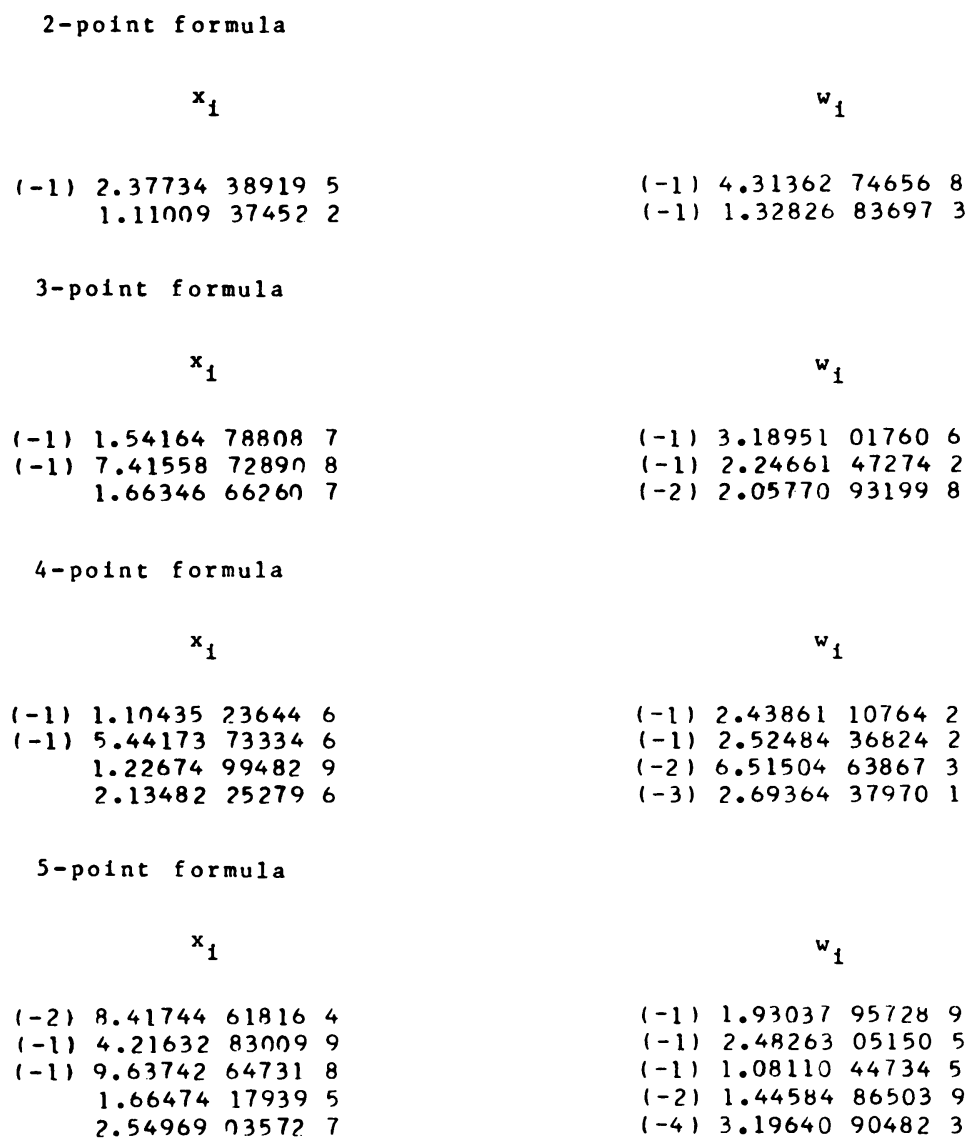


TABLE 3 (continued)

6-point formula

$x_{1}$

$(-2) 6.69431496520$

$(-1) 3.39112560239$

$(-1) 7.84935942750$ 1.36357521996 2. $06126 \quad 692811$ $2.92346 \quad 876713$

7-point formula

$x_{1}$

$(-2) 5.49123050610$

$(-1) 2.80385459369$

$(-1) \quad 6.55644778023$

1.14781304512

1.73629773973

2.42423129794

$3.2658755425 \quad 8$

8-point formula

$x_{1}$

$(-2) \quad 4.61168 \quad 15858 \quad 0$

$(-1) 2.36840207222$

$(-1) 5.58296248923$

$(-1) \quad 9.84661 \quad 141092$

1.49562402012

2.08349433722

2. 76002102163

$3.583413531 n 3$

9-point formula

$x_{1}$

$(-2) \quad 3.94542 \quad 3427395065$

$(-1) 2.035019823397209$

$(-1) 4.82772 \quad 14616 \quad 51686$

$(-1) 8.5703387 n 15 \quad 59237$

1.30815 76394 50268

1.825344245362237

2.408354290471411

$3.07346 \quad 65214 \quad 18331$

$3.88065 \quad 5881282185$

10-point formula

$x_{1}$

$\begin{array}{llll}(-2) & 3.42628 & 01138 \quad 86749\end{array}$

$(-1) 1.77312 \quad 1899441196$

$(-1) \quad 4.2278107835 \quad 69875$

$(-1) \quad 7.5473344456 \quad 28952$

1.157573091138683

$1.6200457105 \quad 40419$

2.13749282650851 ?

$2.71396 \quad 5992426909$

3. $36822 \quad 2166840246$

4. $1609505571 \quad 1724 n$

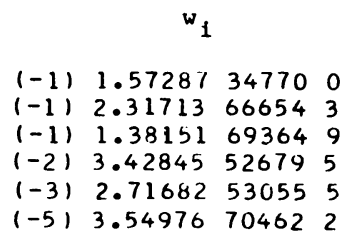

$w_{1}$

$(-1) \quad 1.5728734770 \quad 0$

$(-1) 2.31713666543$

$(-1) 1.38151693649$

$(-2) \quad 3.42845 \quad 52679 \quad 5$

$(-3) \quad 2.7168253055 \quad 5$

$(-5) 3.54976704622$

$$
w_{1}
$$

$(-1) 1.31182570348$ $(-1) \quad 2.11756763142$ $(-1) \quad 1.5518407675 \quad 1$ $(-2) \quad 5.6673424306 \quad 1$ $(-3) \quad 8.93478266391$ $(-4) 4.54210814381$ $(-6) 3.75552259775$

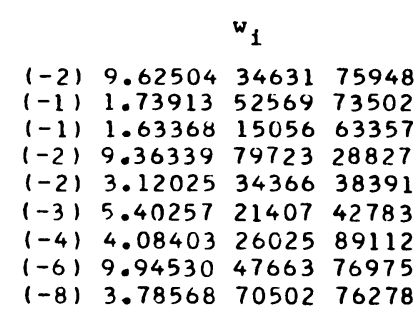

$w_{1}$

$(-2) \quad 8.41668 \quad 63665 \quad 35992$

$(-1) \quad 1.5773780598 \quad 10978$

$(-1) \quad 1.60300 \quad 1505420744$

$(-1) 1.056739696932892$

$(-2) \quad 4.41063 \quad 95402 \quad 82426$

$(-2) \quad 1.07617 \quad 84774 \quad 27428$

$(-3) \quad 1.36559 \quad 1571275019$

$(-5) 7.5673298220 \quad 39683$

$\begin{array}{llll}(-6) & 1.34496 & 57738 & 44262\end{array}$

$(-9) 3.653567136848986$ 
TABLE 3 (continued)

\begin{tabular}{|c|c|c|c|}
\hline \multirow[t]{2}{*}{11} & 1 -point & \multicolumn{2}{|c|}{ formula } \\
\hline & & $x_{1}$ & \\
\hline $\begin{array}{l}(-2) \\
(-1) \\
(-1) \\
(-1)\end{array}$ & $\begin{array}{l}3.01235 \\
1.56296 \\
3.74198 \\
6.71167 \\
1.03398 \\
1.45196 \\
1.91877 \\
2.43357 \\
3.00296 \\
3.64709 \\
4.42682\end{array}$ & $\begin{array}{l}67809 \\
82886 \\
70530 \\
50305 \\
56465 \\
45969 \\
38247 \\
98552 \\
80277 \\
52982 \\
97204\end{array}$ & $\begin{array}{l}91511 \\
65323 \\
56711 \\
64438 \\
21831 \\
91976 \\
88675 \\
23858 \\
35919 \\
26328 \\
46769\end{array}$ \\
\hline & $2-p o 1 n t$ & \multicolumn{2}{|c|}{ formula } \\
\hline & & $x_{1}$ & \\
\hline $\begin{array}{l}(-2) \\
(-1) \\
(-1) \\
(-1) \\
(-1)\end{array}$ & $\begin{array}{l}2.67596 \\
1.39130 \\
3.34211 \\
6.01843 \\
9.30884\end{array}$ & $\begin{array}{r}38529 \\
82411 \\
81025 \\
95516 \\
38305\end{array}$ & $\begin{array}{l}6687 n \\
60834 \\
64531 \\
6234 n \\
86750\end{array}$ \\
\hline-11 & $\begin{array}{l}9.30884 \\
1.31158 \\
1.73714\end{array}$ & $\begin{array}{l}38305 \\
64866 \\
n 4275\end{array}$ & $\begin{array}{l}86750 \\
34759 \\
97650\end{array}$ \\
\hline & $\begin{array}{l}2.20451 \\
2.71527\end{array}$ & $\begin{array}{l}49081 \\
22992\end{array}$ & $\begin{array}{l}77997 \\
95223\end{array}$ \\
\hline & $\begin{array}{l}3.27754 \\
3.91228\end{array}$ & $\begin{array}{l}09264 \\
24599\end{array}$ & $\begin{array}{l}30984 \\
57907\end{array}$ \\
\hline & 4.68026 & 03797 & 3354 \\
\hline
\end{tabular}

$\begin{array}{cccc} & w_{1} \\ (-2) & 7.44055 & 09878 & 89769 \\ (-1) & 1.43487 & 70531 & 10929 \\ (-1) & 1.54963 & 63216 & 15209 \\ (-1) & 1.13709 & 41476 & 46168 \\ (-2) & 5.62965 & 55142 & 88725 \\ (-2) & 1.77366 & 27598 & 88245 \\ (-3) & 3.26453 & 10661 & 40002 \\ (-4) & 3.12389 & 65808 & 86007 \\ (-5) & 1.30439 & 25358 & 55835 \\ (-7) & 1.73694 & 86390 & 16921 \\ (-10) & 3.45407 & 07142 & 18236\end{array}$

$\begin{array}{llll} & w_{1} \\ (-2) & 6.63896 & 49104 & 55207 \\ (-1) & 1.30989 & 46211 & 29896 \\ (-1) & 1.48437 & 89982 & 53906 \\ (-1) & 1.18464 & 93278 & 90080 \\ (-2) & 6.69879 & 22078 & 42853 \\ (-2) & 2.56988 & 20629 & 53840 \\ (-3) & 6.26273 & 84099 & 13355 \\ (-4) & 8.90261 & 21073 & 45358 \\ (-5) & 6.57595 & 07884 & 67420 \\ (-6) & 2.11627 & 18607 & 02677 \\ (-8) & 2.15753 & 71144 & 04678 \\ (-11) & 3.20845 & 69902 & 36600\end{array}$

This check is summarized in Table 1. It gives the relative change of the coefficients of the 12 th-degree polynomial when a relative increase of $3.10^{-33}$ is applied to the moments $\mu_{p}$ used in the computation. The relative change of the zeros and weights is also displayed. This table shows that the recursion scheme (12) is not a stable computation process. However, since a full precision of about 33 digits can be easily obtained in computing the moments $\mu_{p}$ for this particular problem, an error is likely to appear in the 16th place of the computed abscissas and weights for the 12-point formula. For shorter formulas, a better precision is reached.

Though quite general from an algebraic point of view, the method just described is then not obviously applicable to generate high-precision Gaussian rules. For the special case considered here, Table 2 gives the coefficients of the first 12 polynomials orthogonal with respect to the scalar product (4).

The zeros of these polynomials have been computed by means of the Bairstow iteration method [11] and the corresponding weight factors have been deduced. These values are reported in Table 3.

The efficiency of the formula can be checked on the following example

$$
I_{1}=\int_{0}^{\infty} \operatorname{erfc}(x) e^{-\alpha^{2} x^{2}} d x=\frac{\operatorname{arctg} \alpha}{\alpha \sqrt{\pi}}
$$




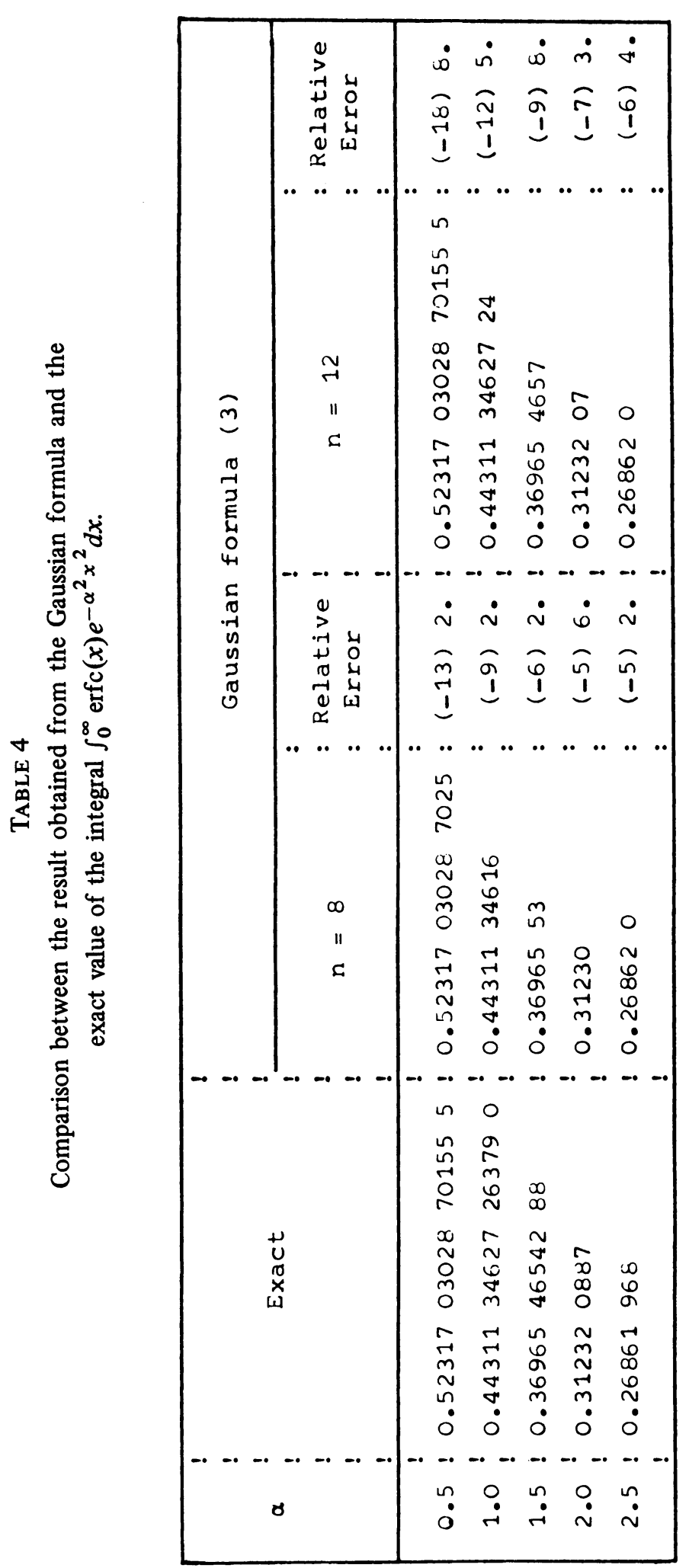


The function $\exp \left(-\alpha^{2} x^{2}\right)$ is easily approximated by a low-degree polynomial, especially when $\alpha$ is small. Table 4 gives some points of comparison between the exact and the approximate integral. It can be seen that a good accuracy (at least 5 places) is readily obtained with the 8-point formula for $\alpha$ less than 2.5 .

Acknowledgments. The authors are very grateful to the Belgian Fonds National de la Recherche Scientifique for financial support.

Institut de Physique, B5

Université de Liège

B-4000 Sart Tilman/Liège 1, Belgium

1. J. H. AHLBERG, E. N. NILSON \& J. L. WALSH, The Theory of Splines and Their Applications, Academic Press, New York, 1967.

2. G. E. FORSYTHE, M. A. MALCOLM \& C. B. MOLER, Computer Methods for Mathematical Computation, Prentice-Hall, Englewood Cliffs, N.J., 1977, p. 92.

3. A. H. STROUD \& D. SECREST, Gaussian Quadrature Formulas, Prentice-Hall, Englewood Cliffs, N.J., 1966.

4. M. ABRAMOWITZ \& I. A. STEGUN, (Eds.), Handbook of Mathematical Functions with Formulas, Graphs and Mathematical Tables, Appl. Math. Series No. 55, U.S. Government Printing Office, Washington, D.C., 1964.

5. PH. LAMBIN \& J. P. VIGNERON, "Tables for the Gaussian computation of $\int_{0}^{\infty} x^{\alpha} \exp (-x) f(x) d x$ for values of $\alpha$ varying continuously between -1 and +1 ," Math. Comp., v. 33, 1979, pp. 805-811.

6. M. M. MORSE \& H. FESHBACH, Methods of Theoretical Physics, McGraw-Hill, New York, 1953, Part I, Chap. 8, p. 928.

7. W. GAUTSCHI, "Construction of Gauss-Christoffel quadrature problems," Math. Comp., v. 22, 1968, pp. $251-270$.

8. W. GAUTSCHI, "On the construction of Gaussian quadrature rules from modified moments," Math. Comp., v. 24, 1970, pp. 245-260.

9. W. GAUTSCHI, "Algorithms 3311 Gaussian quadrature methods," Comm. ACM, v. 11, 1968 , pp. $432-436$.

10. G. H. GOLUB \& J. H. WELSCH, "Calculation of Gauss quadrature rules," Math. Comp., v. 23, 1969, pp. 221-230.

11. J. H. WILKINSON, "The evaluation of the zeros of ill-conditioned polynomials," Parts 1 and 2, Numer. Math., v. 1, 1955, pp. 150-180. 